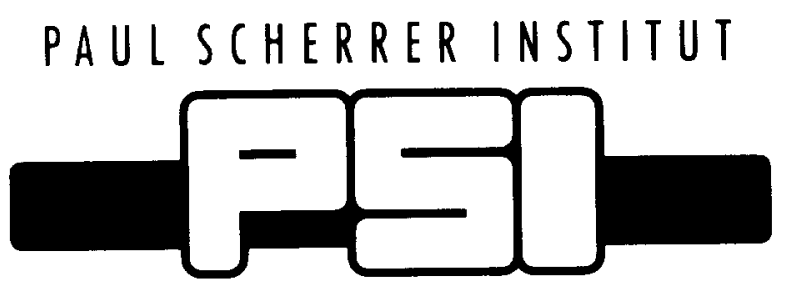

\title{
The Response of Superconductive Films to Localized Energy Deposition
}

\section{A. Zehnder}

Paul Scherrer Institut $\mathrm{CH}-5232$ Villigen PSI, Switzerland
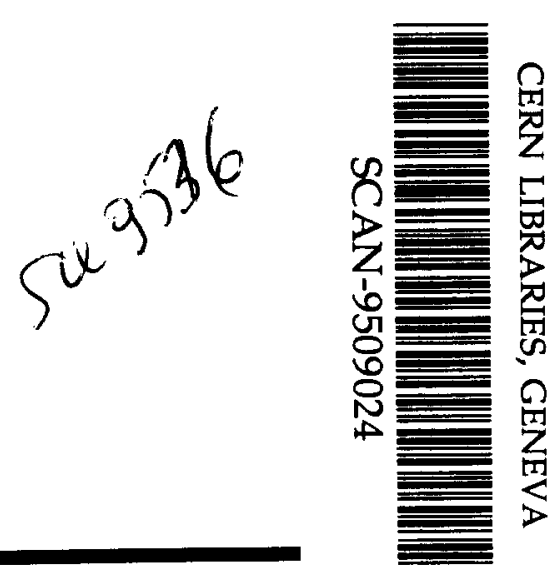


\title{
The Response of Superconductive Films to Localized Energy Deposition
}

\author{
A. Zehnder \\ Paul Scherrer Institut \\ $\mathrm{CH}-5232$ Villigen-PSI \\ Switzerland
}

Superconductive tunneling junctions are promising candidates as detectors for low energy $X$ - ray spectroscopy in particle-, solid-state-, and astrophysics ${ }^{1,2)}$. However, the energy resolution achieved so far deviates from the ultimate resolution calculated on statistical grounds by about one order of magnitude. This discrepancy may be due to the local variation of the relaxation processes following the absorption of the quanta. in this paper we present temperature- and energy-dependent calculations of these processes. It is shown that during the relaxation a substantial amount of the initial energy is converted into phonons with energies less than $2 \Delta_{b}$, where $\Delta_{b}$ is the energy gap of the superconductor in thermal equilibrium at the temperature $T_{b}$. This energy is lost for further production of excess quasiparticles needed for tunneling, and the loss process depends on the quasiparticle and phonon diffusion properties. Its local variation over the junction area limits the energy resolution. In addition the experimentally observed non-linear energy response can also be reproduced by the calculations. The non-linearity stems from the strong self recombination of quasiparticles in the local hot spot. Various superconducting materials were studied in order to select the most suitable one for future superconducting tunneling detectors.

PCAS index: 74.40.k, 74.76.-w

Accepted for publication in Phys.Rev. B, August 1995 


\section{INTRODUCTION}

Energy $E_{0}$ absorbed in a superconductor produces a non-thermal distribution of excess electronic quasiparticles and of phonons. In a voltage-biased superconducting tunneling junction the excess quasiparticles yield a tunneling current through a potential barrier. For a superconductor the energy gap $\Delta_{b} \equiv \Delta\left(T_{\text {bath }}\right)$ is about 1000 times smaller than for a typical semiconductor like $\mathrm{Si}$, hence the number of excess charge carrier $\mathrm{N}_{0}=$ $E_{d} / \Delta_{b}$ should be about 1000 times larger in a superconductor than in a semiconductor. Since the ideal energy resolution $\Delta E / E$ is proportional to $\sqrt{N_{0}} / N_{0}$, a superconducting tunneling junction detector is expected to have a resolution of about $\sqrt{1000} \approx 30$ times better than a semiconductor detector. For $5.89 \mathrm{keV} \mathrm{Mn} \mathrm{K}_{\alpha}$ radiation absorbed in a $\mathrm{Sn} / \mathrm{SnO}_{x} / \mathrm{Sn}$ superconducting tunneling junction detector the resolution should be $4.4 \mathrm{eV}$ FWHM using a Fano factor of 1. However, the experimentally achieved energy resolution amounts to about $50 \mathrm{eV}^{2,3}$, and the collected charge $N_{\text {coll }}$ is less than $40 \%$ of $\mathrm{N}_{\mathrm{o}}$ indicating considerable loss processes. In addition it was experimentally observed by Rothmund and Zehnder ${ }^{4}$ that the collected charge depends in strong non-linear way of the absorbed energy. This non-linearity could not be simulated by calculations done so $\mathrm{far}^{5}$. In these calculations it was assumed that all the quasiparticles were produced with an energy of $\Delta_{b}$ and that the recombination and pair-breaking rates were independent of quasiparticle and phonon energy, and temperature. In addition the scattering processes of quasiparticles and phonons were not included.

Chang and Scalapino ${ }^{6}$ studied the behavior of thin films driven out of equilibrium under spatially uniform steady-state conditions by phonon and quasiparticle tunneling injection. The linearized coupled kinetic equations for the phonon and quasiparticle distribution were solved numerically for different excitation processes. Kurakado ${ }^{7}$ discussed the different relaxation processes and determined $\varepsilon=E_{\circ} / N_{c o l l}$, the mean energy needed to produce one excess quasiparticle, to be about $1.7 \Delta_{0}$ in $\mathrm{Sn}$, implying that only $1 / 1.7=60 \%$ of the initially absorbed energy is converted into excess quasiparticles.

Rothmund ${ }^{8)}$ solved the coupled kinetic equations assuming that the quasiparticles and phonons can be described by a Fermi- and Bose-Einstein distribution function, respectively using temperature- and energy-dependent rates. The effective temperature of the system was calculated time-dependent from the quasiparticle density. 
The aim of the present paper is to derive the number of quasiparticles suitable for tunneling at a later time. It extends the above mentioned treatments in three ways. First we solve the non-linear, coupled kinetic equations in a self consistent way. The energy deposition was assumed to be local and the diffusion of the quasiparticles and phonons were included in the calculations. In addition we include all energy-dependent relaxation processes and finally we apply the results of these calculations to study the behavior of various materials.

\section{RELAXATION PROCESSES}

The temporal behavior of a non-equilibrium system of coupled phonons and quasiparticles was first calculated by Rothwarf and Taylor ${ }^{9}$. They solved the rate equations of quasiparticles at the gap energy $\Delta_{b}$ and of phonons with an energy of $2 \Delta_{b}$. The phonon pair-breaking rate and the temperature-dependent recombination rate were calculated for those fixed excitation energies. These calculations were extended for tunneling junctions including diffusion ${ }^{5}$. However, the superconductor is, after the absorption of the X-ray, highly disturbed. During that stage the energy-dependent relaxation rates for quasiparticles and phonons have to be inserted into the rate equations.

The absorption of radiation with an energy $E_{\circ}$ of a few keV by a superconducting film produces a hot photoelectron of an atomic inner shell with an energy of $E_{0}-E_{B}$, where $E_{B}$ is the binding energy of the shell. This electron ionizes atoms along its trajectory. The remaining hole in the inner shell of the initially ionized atom is filled up again by radiative transitions (X-rays) or by Auger processes. These secondary transitions are mostly absorbed in the film and contribute to the so-called photo peak. However, the $X$ ray can also escape from the film, leading to a well-defined deficiency in the deposited energy and generating the so-called escape lines. We used these lines to determine the non-linear relation between the absorbed energy and collected charge ${ }^{4}$.

The main relaxation process of the primary and secondary electrons from the ionized atoms proceeds through electron- electron interaction so long as the electron energy is larger than a characteristic energy $\omega_{c} \equiv\left(E_{F} E_{D}\right)^{1 / 2} . E_{F}$ and $E_{D}$ are the usual Fermi and Debye energies, respectively (throughout the paper $\hbar=1$ is assumed). Electron-phonon interaction become dominant at lower energies ${ }^{6)}$. Thus after a few collisions the elec- 
trons drops below $\omega_{c}$ and it decays further by emitting phonons. In the average $\sqrt{E_{F} / E_{D}}>15$ phonons will be emitted before the electron drops into states of energy smaller than the Debye energy ${ }^{6)}$. Further de-excitation proceeds primarily through inelastic scattering with phonon emission. These phonons are subject to various processes: they can be absorbed by the Cooper pair system creating excited quasiparticles, undergo an anharmonic decay process into two phonons, or inelastic scatter thus exciting quasiparticles. Finally they may also escape into the substrate. After a few nanoseconds an excess population of quasiparticles near the band gap and of subgap phonons is generated. The system returns to the initial state of equilibrium (bath temperature) mainly by recombination of quasiparticles to Cooper-pairs, by escape of phonons into the substrate, and by tunneling in case of a superconducting tunneling device.

At low temperatures $\left(T / T_{C} \sim 0.1\right)$ the recombination time is of the order of milliseconds. In a superconducting tunneling junction, however, the tunneling time for a quasiparticle is of the order of $\mu \mathrm{sec}$. Therefore excess quasiparticles relax through tunneling. In the present paper we only show the results of the de-excitation processes in a superconducting film and do not include the tunnel process.

\section{GENERALIZED ROTHWARF- TAYLOR EQUATIONS}

To derive the number of quasiparticles and phonons and their energy distribution at the end of the relaxation cascade, we have to solve a set of coupled rate equations for quasiparticles and phonons. As usual we take the zero energy level at the Fermi energy $E_{F}$. The rate equations for the number of excess quasiparticles and phonons with energy $\omega$ and $\Omega$ are:

$$
\begin{aligned}
\frac{d Q(\omega)}{d t} & =\int_{0}^{\infty} d \Omega\left[\gamma_{\text {phe }}(\omega+\Omega, \omega) \cdot Q(\omega+\Omega)-\gamma_{\text {pha }}(\omega, \omega+\Omega) \cdot\right] \\
& \left.+\int_{0}^{\omega-\Delta} d \Omega\left[\gamma_{\text {pha }}(\omega-\Omega, \omega)\right) \cdot Q(\omega-\Omega)-\gamma_{\text {phe }}(\omega, \omega-\Omega) \cdot Q(\omega)\right] \\
& +\int_{\omega+\Delta}^{\infty} d \Omega\left[\gamma_{p b}(\omega, \Omega-\omega) \cdot P(\Omega)-\gamma_{\text {rec }}(\omega, \Omega-\omega) \cdot \frac{Q(\omega) \cdot Q(\Omega-\omega)}{4 \cdot N_{0} \cdot V(t)}\right]
\end{aligned}
$$




$$
\begin{aligned}
\frac{d P_{F}(\Omega)}{d t}= & \int_{0}^{\infty} d \omega\left[\gamma_{\text {phe }}(\omega+\Omega, \omega) \cdot Q(\omega+\Omega) \cdot \Theta(\omega+\Omega-\Delta)-\gamma_{\text {pha }}(\omega, \omega+\Omega) \cdot Q(\omega) \cdot \Theta(\omega-\Delta)\right] \\
& +\int_{\omega+\Delta}^{\infty} d \omega\left[\gamma_{r e c}(\omega, \Omega-\omega) \cdot \frac{Q(\omega) \cdot Q(\Omega-\omega)}{8 \cdot N_{0} \cdot V(t)}-\gamma_{p b}(\omega, \Omega-\omega) \cdot P_{F}(\Omega) / 2\right] \\
& -\int_{0}^{\Omega} d \Omega^{\prime} \cdot \gamma_{\text {anh }}\left(\Omega, \Omega^{\prime}\right) \cdot P_{F}(\Omega) / 2+\int_{\Omega}^{E_{0}} d \Omega^{\prime} \cdot \gamma_{\text {anh }}\left(\Omega^{\prime}, \Omega\right) \cdot P_{F}\left(\Omega^{\prime}\right)-\Gamma_{\text {esc }} \cdot P_{F}(\Omega)
\end{aligned}
$$

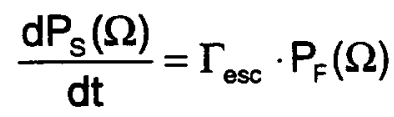

Eq. (1) and (2) are derived from the Eq. $1 \mathrm{a}$ and $1 \mathrm{~b}$ of the paper by Chang and Scalapino ${ }^{6)}$ by using the identities

$Q(\omega) \equiv 4 \cdot N(0) \cdot V(t) \cdot \rho(\omega) \cdot f(\omega)$

and

$P(\Omega) \equiv 2 \cdot N \cdot V(t) \cdot F(\Omega) \cdot n(\Omega)$

The equation (1), (2) and (3) conserve the total energy of the system. The steady-state functions for the quasiparticle and phonon distributions are $f(\omega)$ and $n(\Omega)$, respectively. The function $\rho(\omega)$ is the normalized quasiparticle density of states given by

$$
\rho(\omega)=\frac{\omega}{\sqrt{\omega^{2}-\Delta^{2}}} \cdot \Theta(\omega-\Delta)
$$

where $\Theta(x)=1$ for $x>0$ and 0 for $x<0 . F(\Omega)$ is the phonon density of states per ion. $F(\Omega)$ can be obtained from neutron-scattering measurements, but here we use the Debye model ${ }^{\left.10,{ }^{11}\right)}$ for phonons so that

$$
F(\Omega)= \begin{cases}9 \cdot \frac{\Omega^{2}}{E_{D}^{3}} & \text { for } \Omega \leq E_{D} \\ 0 & \text { for } \Omega>E_{D}\end{cases}
$$

$\mathrm{N}$ is the number of ions per unit volume and $N(0)$ the usual single-spin band and Cou- 
lomb-dressed electron density of states at the Fermi energy.

$Q(\omega)$ and $\left.P_{F}(\Omega)\right)$ are the numbers of quasiparticles and phonons in the film with their corresponding energies $\omega>\Delta$ and $\Omega>0$, respectively. $\Delta$ is the effective energy gap computed from the quasiparticle distribution $f(\omega)$, as shown below. $P_{s}(\Omega)$ is the number density of phonons in the substrate. In Eq. (1), the rate $\gamma_{\text {phe }}$ (see Eq. (8)) describes the inelastic scattering of quasiparticles with emission of phonons with energy $\Omega$ and the rate $\gamma_{\text {pha }}$ (see Eq. (9)) the excitation of quasiparticles via absorption of phonons with energy $\Omega$, respectively. The rate $\gamma_{p b}$ (see Eq. (10)) represents the breakup of pairs by phonons of the energy $\Omega$. Here two quasiparticles with $\omega$ and $\omega-\Omega$ are created and a phonon with energy $\Omega$ is absorbed. The rate $\gamma_{r e c}$ (see Eq. (11)) stands for the recombination of two quasiparticles with energies $\omega$ and $\Omega$ - $\omega$ into a Cooper-pair and the emission of a phonon with energy $\Omega$. This important term is quadratic in the number density $Q(\omega) / 4 N(0) V(t)$ of the quasiparticles and determines the non-linear behavior as well as part of the losses. The hot spot volume $V(t)$ (see Eq. (17)) is responsible for the dynamical behavior. It is discussed in detail below.

The rate equation for phonons is given by Eq. (2). It contains the corresponding phonon creation/destruction rates $\gamma_{\text {phe }}, \gamma_{\text {pha }}$ of Eq. (1). The factor 2 included in the rate $\gamma_{\text {rec }}$ and $\gamma_{p b}$ reflects the fact that two quasiparticles are involved in the recombination and pair break-up processes and only one phonon. In addition, Eq. (2) contains a term for the anharmonic decay of phonons of the energy $\Omega$ into two phonons of the energies $\Omega^{\prime}$ and $\Omega-\Omega^{\prime}$ with the rate $\gamma_{\text {anh }}\left(\Omega, \Omega^{\prime}\right)$ (see Eq. (12)). The last term depicts the escape rate $\Gamma_{\text {esc }}$ (see Eq. (13)) of phonons from the film into the substrate.

The energy-dependent rates in Eq. (1) and Eq. (2) are derived from Chang and Scalapino ${ }^{6}$ using their Eqs. (4) and (5):

$$
\begin{aligned}
& \gamma_{\text {phe }}\left(E, E^{\prime}\right)=\frac{2 \pi \alpha^{2}}{\hbar N} \cdot\left(1-\frac{\Delta^{2}}{E \cdot E^{\prime}}\right) \cdot\left(\frac{P_{F}(E)}{V(t)}+N \cdot F(E)\right) \cdot\left(\rho\left(E^{\prime}\right)-\frac{Q\left(E^{\prime}\right)}{4 \cdot N(0) \cdot V(t)}\right) \\
& \gamma_{\text {pha }}\left(E, E^{\prime}\right)=\frac{2 \pi \alpha^{2}}{\hbar N} \cdot\left(1-\frac{\Delta^{2}}{E \cdot E^{\prime}}\right) \cdot \frac{P_{F}(E)}{V(t)} \cdot\left(\rho\left(E^{\prime}\right)-\frac{Q\left(E^{\prime}\right)}{4 \cdot N(0) \cdot V(t)}\right)
\end{aligned}
$$




$$
\begin{aligned}
& \gamma_{p b}\left(E, E^{\prime}\right)=\frac{8 \pi \alpha^{2} \cdot N(0)}{\hbar N} \cdot\left(1+\frac{\Delta^{2}}{E \cdot E^{\prime}}\right) \cdot\left(\rho(E)-\frac{Q(E)}{4 \cdot N(0) \cdot V(t)}\right) \cdot\left(\rho\left(E^{\prime}\right)-\frac{Q\left(E^{\prime}\right)}{4 \cdot N(0) \cdot V(t)}\right) \\
& \gamma_{\text {rec }}\left(E, E^{\prime}\right)=\frac{2 \pi \alpha^{2}}{\hbar N} \cdot\left(1+\frac{\Delta^{2}}{E \cdot E^{\prime}}\right) \cdot\left(\frac{P_{F}(E)}{V(t)}+N \cdot F(E)\right) \\
& \gamma_{\text {anh }}\left(E, E^{\prime}\right)=\frac{30 \cdot\left(E^{\prime 2}\left(E-E^{\prime}\right)^{2}\right)}{\left(E_{D(S i)}\right)^{2}} \\
& \Gamma_{\text {esc }}=\frac{\alpha_{t} c}{4 h}
\end{aligned}
$$

The rates $\gamma_{\mathrm{phe}}, \gamma_{\mathrm{pha}}, \gamma_{\mathrm{rec}}$, and $\gamma_{\mathrm{pb}}$ depend on the energy distribution of the time-dependent densities of quasiparticle and phonon $Q(\omega) / 4 N(0) V(t)$ and $P_{F}(\Omega) / N V(t)$, respectively. Therefore, the Rothwarf-Taylor equations are solved in a fully self-consistent way without the linearization used by Chang and Scalapino ${ }^{6)}$. The parameter $\alpha^{2}$ can be obtained by using neutron scattering data to find $F(\Omega)$ and then dividing this $F(\Omega)$ out of the $\alpha^{2}(\Omega) F(\Omega)$ obtained from tunneling experiments. Here, however, following Kaplan et. al. ${ }^{11}$, we use the Debye model to determine $\alpha^{2}$. The energy distribution of the anharmonic decay rate $\gamma_{\text {anh }}\left(\Omega, \Omega^{\prime}\right)$ is taken from Maris ${ }^{12)}$. Since the total anharmonic decay rate is not known for the materials of interest, it is scaled to the known Si rate using the Debye temperature as a scale factor ${ }^{12}$. The escape rate is given via the phonon transmission probability $\alpha_{t}$ through the interface between the film and the substrate, $\mathrm{c}$ is the mean phonon velocity and $\mathrm{h}$ is the thickness of the film.

\section{A. Diffusion}

The time-dependent volume $V(t)$ of the hot spot in the thin film is calculated, using a two- dimensional diffusion model, in the following way: The differential diffusion length is given by $\delta s_{i}^{2}=N_{i}\left(v_{i x}^{2}+v_{i y}^{2}\right) \tau_{i}^{2}=N_{i} v_{i}^{2} \tau_{i}^{2} / 2$, (i= qp or $p h$ ) where $N_{i}$ is the number of collisions given by $N_{i}=\delta t /\left(\tau_{i} / w_{i}\right)$. The mean scattering rates $\tau_{i}^{-1}$ for quasiparticles and phonons, respectively, are given by: 
$\tau_{\mathrm{qp}}^{-1}(\omega, T)=\tau_{\mathrm{qp} \text { kap }}^{-1}(\omega, T)+\mathrm{v}_{\mathrm{qp}} / \lambda$

$\tau_{\mathrm{ph}}^{-1}(\Omega, T)=\tau_{\mathrm{ph}}^{-1}(\Omega, T)+\mathrm{c} / \lambda$

The total lifetime $\tau_{i \text { kap }}$ is calculated according to Kaplan et al. ${ }^{11)}$ for quasiparticles and phonons at their respective temperature and energy. The quasiparticle velocity $v_{\mathrm{qp}}$ is

calculated from the Fermi velocity $v_{F}$ and approximated by ${ }^{13)} v_{\Phi p}=\left\langle v^{2}\right\rangle^{1 / 2}=v_{F} \sqrt{T / \Delta}$. It was assumed that the phonons propagate with sound velocity $c$. The quasiparticle mean free path $\lambda$ can be determined from the resistivity of the film, but for the present calculations it is a free parameter. The weight factors $w_{i}$ are calculated from the total energies $\omega_{\text {ot }}(t)$ and $\Omega_{\text {tot }}(t)$ stored in the quasiparticle and the phonon system, respectively:

$w_{\mathbf{p}}=\frac{\omega_{\text {tot }}}{\omega_{\text {tot }}+\Omega_{\text {tot }}}$

$w_{p h}=\frac{\Omega_{t a}}{\omega_{t o t}+\Omega_{t o t}}$.

The diffusion length for the combined quasiparticie-phonon system is approximated by $\delta s^{2}=\delta s_{\mathrm{qp}}^{2}+\delta s_{\mathrm{ph}}^{2}$. Therefore the diffusion length $s$ at time $t$ is given by:

$$
s^{2}(t)=\int_{t_{0}}^{t} d t\left\{v_{q p}^{2} w_{q p} \tau_{q p}(\bar{\omega})+c^{2} w_{p h} \tau_{p h}(\bar{\Omega})\right\} / 2 .
$$

The weight factors $w_{i}$ reflects the fact that at early time with phonon domination, the hot spot diffusion is much slower than at latter time where the quasiparticles dominate.

The corresponding hot spot volume $V(t)$ is finally given by:

$$
V(t)=\pi h s^{2}(t)+V_{0}
$$

where $V_{0}$ is the initial volume determined by the range of the photoelectron, it is typically $0.1 \mu \mathrm{m}^{3}$. $V_{0}$ also depends on the nature of the incident radiation. However, the calcula- 
tions below showed little influence on $V_{0}$ mainly because $V(t)-V_{0}$ gets quickly larger than $V_{0}$ in about a 1 psec., a time scale where the losses are still small. Eq. (16) is only an approximation; the exact solution leads to a differential equation and is discussed in detail in Ref. 13.

\section{B. Temperature Distribution}

From the assumed Gaussian quasiparticle density $q(t, r)$ we determine the effective quasiparticle temperature at a given distance $r$ from the locus of absorption of the $X$-ray at a time $\mathrm{t}$ :

$q(t, r)=q_{0}(t) \cdot e^{-\frac{r^{2}}{2 s^{2}}}$

where the quasiparticle density $q_{0}$ is defined by

$q_{0}(t)=\int_{\Delta}^{\infty} d \omega \cdot \frac{Q(\omega, t)}{V}$

and the variance $s(t)$ and the hot-spot volume $V(t)$ by Eqs. (16) and (17) respectively. The density $q(t, r)$ is then equaled to the number density $q_{t h}(T, r)$ of the thermal quasiparticles:

$$
\begin{aligned}
q_{t h}(T, r) & =4 N(0) \cdot \int_{\Delta}^{\infty} \rho(\omega) /\left(1+e^{\omega / k T}\right) \cdot d \omega \\
& =4 N(0) \cdot \Delta \cdot \sum_{n=1}^{\infty}(-1)^{n+1} \cdot K_{1}(n \cdot \Delta / k T)
\end{aligned}
$$

where $K_{1}(z)$ is the modified Bessel function ${ }^{14)}$. The widely used equation $\mathrm{q}_{\mathrm{th}}(\mathrm{T}) \approx 2 \mathrm{~N}(0) \sqrt{2 \pi \Delta \cdot \mathrm{kT}} \mathrm{e}^{-\frac{\Delta_{0}}{\mathrm{kT}}}$ is an approximation of Eq. (19) for $\mathrm{n}=1$. Near $\mathrm{T}_{\mathrm{c}}$ it is incorrect up to $32 \%$, while at $0.1 \mathrm{~T}_{\mathrm{c}}$ up to $8 \%$.

The temperature distribution $T(t, r)$ was calculated from Eqs. (18) and (19). However, to simplify the numerical solution of Eqs. (1) and (2) a mean temperature $T_{m}(t)$ was computed as follows ( $T_{m}$ hence abbreviate $\left.T\right)$ : 
$T_{m}(t)=\frac{1}{2 s^{2}} \int_{r_{c}}^{\infty} T(t, r) \cdot e^{-\frac{r^{2}}{2 s^{2}}} \cdot r \cdot d r+\left(1-e^{-\frac{r_{c}^{2}}{2 s^{2}}}\right) \cdot T_{C}$

The critical radius $r_{c}(t)$ is defined as the radius where $q_{c}\left(r_{c}\right)=q_{t h}\left(T_{c}\right)$ and is given by

$r_{c}=\sqrt{-2 \cdot s^{2} \cdot \ln \left(q_{c} / q_{0}\right)}$

For quasiparticles in equilibrium described by the Fermi function $f(\omega, T)$, the temperature dependence of the energy gap in thermal equilibrium is $\Delta(T)=\Delta_{0} \cdot \sqrt{1-T / T_{C}} \cdot \ln$ the presence of a non-thermal quasiparticle distribution $f(\omega)=Q(\omega) / 4 N(0) \rho(\omega) V(t)$ the gap is modified and called $\Delta . \delta \Delta$ is the change of the energy gap $\Delta-\Delta(T)$ and can be calculated within a linear approximation given by Chang and Scalapino ${ }^{6)}$ :

$$
\delta \Delta=\Delta(T) \frac{\int_{\Delta(T)}^{\infty} d \omega \cdot \frac{2(f(\omega)-f(\omega, T))}{\sqrt{\omega^{2}-\Delta^{2}(T)}}}{1-\int_{\Delta(T)}^{\infty} d \omega \cdot \rho(\omega) \cdot e^{\omega / k T \cdot f^{2}(\omega, T) / k T}}
$$

For the present calculations the effective gap $\Delta=\Delta(T)+\delta \Delta$ is used.

\section{NUMERICAL SOLUTION}

Equations (1), (2) and (3) represent a system of coupled non-linear differential equations which can be integrated numerically. In the present work, the integrals were replaced by summations over $n$ intervals from $0 \mathrm{eV}$ to the Debye energy $E_{D}$ for quasiparticle and phonon energies. The system of differential equations was solved on a vector processor (VAX 9000) using a numerical differential equation solving routine ${ }^{15}$. The calculations were checked by summing up the energies of all quasiparticles and phonons. If the rate equations are solved correctly, the total energy must be constant. The accuracy was checked by increasing the summation intervals. It was found that between 200 and 400 intervals no significant change of the solution $Q(\omega, t), P_{F}(\Omega, t)$, and 
$P_{S}(\Omega, t)$ takes place. Therefore 200 intervals were used for the calculations below.

The solutions $Q(\omega, t), P_{F}(\Omega, t)$ and $P_{S}(\Omega, t)$ are the time- and energy-dependent occupation numbers of quasiparticles and phonons in the film and in the substrate. The starting condition was set to $t_{0}=0.01$ psec. The initial phonons-to-quasiparticles ratio was assumed to be $R=\sqrt{E_{F} / E_{D}}$ (see sect. II). All initial phonons and quasiparticles were located at the Debye energy. This assumption was not critical, since in a very short time (<1psec.) the quasiparticles and phonons are down converted (see section V). The material-dependent values are given in Tab. 1, and are, if not otherwise stated, from Ref. ${ }^{11)}$ and from references therein. All calculations were made with a phonon transmissivity to the substrate of $\alpha_{4}=0.3$, for a film with a surface dimension of 50 by $50 \mathrm{~m} \mu^{2}$ and a film thickness of $0.5 \mu \mathrm{m}$.

\section{DISCUSSION}

The non-linearity and reduced energy resolution may be due to self-recombination of the quasiparticles in the following way: The self-recombination rate term in Eqs. 1 and 2 is quadratic in the number density $Q(\omega)$ and is proportional to $1 N(t)$, therefore, the quasiparticle density $Q(\omega) N(t)$ is responsible for the non-linear energy behavior. The mean free path $\lambda$ of the quasiparticle depends on the material and structure of the films. Hence so does $V(t)$. Most of the superconducting tunneling junctions produced so far were polycrystalline films with rather small mean free paths of the quasiparticles.

\section{A. Loss Processes and finite Resolution}

After the absorption of an X-ray photon in a time less than 0.01 psec. a typical hot spot volume of $0.1 \mu \mathrm{m}^{3}$ is formed with a high energy density. This volume is much smaller than the junction volume of a typical $1250 \mu \mathrm{m}^{3}$. The temperature $T$ in the hot spot volume is elevated compared to the bath temperature $T_{b}$ and hence the actual superconducting energy gap $\Delta$ is reduced in comparison to $\Delta_{b}$. For a very high energy density supercritical temperatures are obtained and superconductivity is locally destroyed. Separate experiments have demonstrated that a normal state hot spot is created in Al and $\mathrm{Nb}$ films after the absorption of a $5.89 \mathrm{keV} \mathrm{X}$-ray ${ }^{16)}$ and equally so in $\mathrm{Ta}, \mathrm{Nb}$, and 
Sn films after the deposition of about $150 \mathrm{keV}$ by an alpha particle ${ }^{17}$. As a result of the local energy gap suppression, a fraction of the initial energy gets lost, mainly through the production of phonons with energies of less than $2 \Delta_{b}$. The main phonon-related loss mechanisms are:

- Phonons with energies less than $2 \Delta_{b}$ are created by phonon emission through quasiparticle scattering and by anharmonic decay processes of phonons.

- Phonons in the energy range of $2 \Delta$ and $2 \Delta_{b}$ are lost for the pair breaking mechanism if they leave the hot spot. Their energy is then too small to create 2 quasiparticles with an energy $>\Delta_{b}$ by Cooper pair breaking. The probability of their being absorbed in the hot spot region is given by $e^{-s / \lambda}$ ph , where $s$ is the hot spot radius given by Eq. (16) and $\lambda_{p h}=c \tau_{\text {ph }}$ (see Eq. (14)) is the phonon mean free path.

- A non-zero probability exists for phonons of all energies to escape from the film into the substrate before breaking up a Cooper pair. Unless they scatter back into the film, still with energy $>2 \Delta_{b}$, such phonons are lost for Cooper pair breaking.

A further loss mechanism involves quasiparticles:

- Quasiparticles in the hot spot can relax to energies near the gap when the gap $\Delta$ is still reduced compared to $\Delta_{b}$. If they are not subsequently excited by phonon absorption to energies above $\Delta_{b}$, they will condense to Cooper pairs while the temperature of the hot spot relaxes to the bath temperature $T_{b}$.

For the further discussion we define the quantities $E_{>}=E_{q p>}+E_{p h>}$ and $E_{<}=E_{q p<}+E_{p h<}$ given by: 


$$
\begin{array}{rlr}
E_{\mathrm{qp}>} & =\int_{\Delta}^{\infty} \omega \cdot \mathrm{Q}(\omega) \mathrm{d} \omega ; & E_{\mathrm{ph}>}=\int_{2 \Delta}^{\infty} \Omega \cdot \mathrm{P}_{\mathrm{F}}(\Omega) \mathrm{d} \Omega \\
E_{\mathrm{qp}<} & =\int_{0}^{\Delta} \omega \cdot \mathrm{Q}(\omega) \mathrm{d} \omega ; & E_{\mathrm{ph}<}=\int_{0}^{2 \Delta} \Omega \cdot \mathrm{P}_{\mathrm{F}}(\Omega) \mathrm{d} \Omega \\
\mathrm{E}_{\mathrm{S}} & =\int_{0}^{\infty} \Omega \cdot \mathrm{P}_{\mathrm{S}}(\Omega) \mathrm{d} \Omega
\end{array}
$$

Phonons and quasiparticles with energy $E_{>}$may contribute to the tunneling current at a later time. The remaining subgap phonons and quasiparticles with energies $E_{<}$are lost as contributors to the tunneling current. $E_{s}$ is the energy stored in the phonons escaping to the substrate; it is also lost. A high-resolution superconducting junction detector requires that the largest possible fraction of the initially deposited energy $E_{0}$ is stored in E.

In contrast to superconducting tunneling junction devices, $\mathrm{X}$-ray absorbers of bolometers are optimized by converting the initial energy into subgap phonons as rapidly as possible. Thus bolometers should minimize $E_{>}$. Results on experiments using superconductors as absorbers on bolometers were reported by Labov et al. ${ }^{18)}$ and Stahle et $\mathrm{al}^{19)}$. After the absorption of the $\mathrm{X}$-ray, a phonon signal demonstrating two different time scales was observed: a prompt one that can be attributed to subgap phonons produced in the hot-spot, and a slow one that can be related to the recombination phonons of quasiparticles.

\section{B. Energy Distribution of Quasiparticles and Phonons as a Function of Time}

Fig. 1 shows the quasiparticle and phonon energy distributions in Sn for different times after the absorption of a $5.89 \mathrm{keV} \mathrm{Mn} \mathrm{X-ray.} \mathrm{The} \mathrm{distributions} \mathrm{are} \mathrm{obtained} \mathrm{from} \mathrm{the} \mathrm{cal-}$ culation described in section III and IV.

A rapid conversion of the highly excited quasiparticles into phonons is observed (see Fig. 1a) on a time scale of less than 1 psec. corresponding to the scattering time $\tau_{\text {phe }}$ (Eq. 8) of quasiparticles with phonon emission. Within about 10 psec. the energy of phonons is reduced by anharmonic processes (see Fig. $1 \mathrm{~b}$ and c). From 10 to 100 
psec. the breaking of Cooper pairs by phonons producing quasiparticles is the dominant relaxation process (see Eq. 10). The population of quasiparticles near the gap and of phonons in the film and in the substrate are displayed in Fig. $1 \mathrm{~d}$ at $2.5 \mathrm{nsec}$. Most of the phonons have now energies less than $2 \Delta_{b}$, and the escape of these phonons into the substrate is now the main loss process.

Fig. 2 shows the time evolution between 0.1 psec. and 10 nsec. of the energy $E_{q p>}, E_{p h>}$ $E_{p h<}$, and $E_{S}$ (see Eq. 23) after the absorption of a $5.89 \mathrm{keV} X$-ray. The calculations were made for quasiparticle mean free paths of 10 and $200 \mathrm{~nm}$; the results show a marked differences. A shorter mean free path causes a larger fraction of the initial energy to be converted into phonons; this leads to inevitable loss processes. Therefore $E_{q p>}$ is smaller for shorter mean free paths. The plots are arranged in the order of decreasing $E_{q p>}$, which scales roughly as the ratio $E_{D} / \Delta_{b}$. It is about 360 for $A l$ and only 14 for $\mathrm{Pb}$. Due to the small Debye energy and the large energy gab in $\mathrm{Pb}$, most of the phonons produced during relaxation are subgab phonons and therefore lost.

\section{Dependence on Material}

We calculated $E_{>}$as a function of the mean free path and of different materials. The parameters for the different superconductors were taken from Ref. 11.

Fig. 3 shows the result of the calculations. There exists a group of materials $(\mathrm{V}, \mathrm{Ta}, \mathrm{Nb}$ and $S n$ ) with a typical $E_{>}$of about 40 to $60 \%$ of the initial energy. This value agrees well with the widely used value of $\varepsilon=1.7 \Delta_{0}$ given by Ref. 7 . However, materials with a short pair-breaking time $\tau_{p b}$ (e.g. $\mathrm{Pb}$ ) show larger losses and are therefore not ideal for $X$-ray absorbers in superconducting tunneling junctions. They may be good absorbers for bolometric detectors, since a large fraction of the initial energy is converted quickly into subgap phonons.

Due to the long characteristic time $\tau_{0}$ in Aluminum, $X$-ray absorbed in Al films displays little losses, mainly because the absorbed energy is confined in the excited phonons. However, the phonons losses into the substrates are important in thin Al films. For strongly disturbed films with $\lambda<20 \mathrm{~nm}$ the phonon losses are smaller then with larger mean free paths and therefore $E_{>}$is larger. 


\section{Non-linear Energy Response}

In earlier works superconducting tunneling junctions made of Sn with different granularity were extensively investigated by us $^{4), 20)}$. It was experimentally shown that the junctions have a non-linear energy response and that non-linearity anticorrelates with the amount of the collected charge. Subsequently the Rothwarf-Taylor equations were solved, but the experimentally observed non-linearity could not be simulated ${ }^{5}$.

For the present work $\mathrm{E}_{>}$(see Eq. (23)) was calculated for $\mathrm{Sn}$ and $\mathrm{Nb}$ in function of the absorbed energy $E_{0}$ and of the mean free path, respectively. The results are shown in Fig. 4 and 5. A pronounced non-linearity is clearly visible. A quadratic least-square fit was applied to describe the functional dependence of the $E_{>}$and the absorbed energy $E_{0}$. The results are shown as inserts in the figures. One notes that in the case of $\mathrm{Nb}$ the non-linearity decreases, as expected, for longer mean free path, whereas in Sn a maximum of the non-linearity is reached at about $120 \mathrm{~nm}$ mean free path.

Experimentally we determined the non-linearity using the so-called escape lines of the Mn-X-rays, explained in section II. Since the experimental data are only published in proceedings, a short explanation of the method used is given below:

For the present comparison of the experimental data with the calculations, we define the non-linearity as the deviation from a linear energy relation between the theoretical $\left(E_{\text {theory }}\right)$ and the measured energies $\left(E_{\text {meas }}=\Delta_{b} Q_{\text {meas }}\right) . Q_{\text {meas }}$ is calculated from pulser signals of known charge input that were recorded simultaneously with the $X$-rays. The pulse height and rise time of the $\mathrm{X}$-ray and pulser signals were recorded in an event-byevent mode. Note that, owing to the finite RC-time constant of the amplifier circuits, a different rise time of the signal results in a different factor of the pulse-height-to-charge conversion. This effect can produce an apparent non-linearity. The $\mathrm{X}$-ray spectra were generated by off-line analysis using the known pulse-height-to-charge conversion factors for the corresponding measured $\mathrm{X}$-ray rise time.

$X$-ray peaks resulting from the upper film of the junction showed little variation of the rise time of the energies of the photo and escape peaks. Therefore only $X$-rays absorbed in the upper film were taken for the non-linearity analysis. In addition, the linearity of the total electronic chain was tested using a highly linear pulser with different rise times.

Since an energy bias introduced by off-sets in the electronic chain can not be excluded 
a priori, we made a quadratic fit to the five most prominent escape $X$-ray lines $L_{i}$. viz.

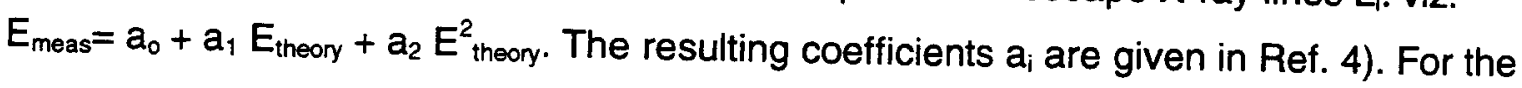
present work non-linearity is defined as follows:

$$
n\left(L_{i}\right)=\frac{E_{\text {meas }}\left(K_{\alpha}\right)-a_{0}}{E_{\text {theory }}\left(K_{\alpha}\right)} \cdot E_{\text {theory }}\left(L_{i}\right)-\left(E_{\text {meas }}\left(L_{i}\right)-a_{o}\right)
$$

We evaluated a total of $15 \mathrm{Sn} / \mathrm{SnO} \mathrm{X}_{x} / \mathrm{Sn}$ junctions. Three results of typical junctions, together with recent data from Verhoeve et. $\mathrm{al}^{21)}$ for $\mathrm{Nb}$ junctions are given in Tab. 2. The PSI junctions had a surface area of 50 by $50 \mu \mathrm{m}^{2}$, wheras the ESTEC junctions of 20 by $20 \mu \mathrm{m}^{2}$. The film thickness and granularity varied, therefore the length of their mean fiee path is different. Also included in Tab 2 are the results of our calculations for three different mean free paths for the PSI Sn junctions. The agreement between the experiment and the calculations is qualitatively satisfactory. A more detailed comparison including the model for the tunneling junction device and the charge-sensitive preamplifier transfer function will be published elsewhere.

\section{SUMMARY}

The present calculations show that a fraction of the initially deposited energy in superconducting tunneling junctions is converted into subgap phonons and is therefore lost for subsequent tunneling and hence for the charge collection. These substantial losses depend on the quasiparticle and phonon mean free paths. They may vary over the junction area, owing to spatial inhomogeneities, the granularity, and /or the impurities of the superconducting film. Therefore, the non-ideal energy resolution and incomplete charge collection observed can be explained in terms of the variation of the loss processes. Future works will have to concentrate on the fabrication of epitaxial films to be used as X-ray absorbers of high spatial homogeneity.

\section{ACKNOWLEDGMENTS}

The present work benefited from useful discussions with W. Rothmund, H. R. Ott, Ph. Lerch, F. Finkbeiner Zhou Shiping, and D. van Vechten, whom we thank. The extensive calculations were supported by the PSI computing division. The work was financially 
supported by the a special grant of the ETH Zurich and by the Swiss KWF Program. 


\section{REFERENCES}

1) A. Barone, R. Cristiano, and S. Pagano eds. Proceedings. of the Workshop on $X$ Ray Detection by Superconducting Tunnel Junctions, (World Scientific Publishing Co. Singapore 1991).

2) A. Zehnder, in Festi-Val. Festschrift for Val Telegdi. Essays in physics in honor of his 65th birthday, Publisher: Elsevier Science Publishers B.V., and North-Holland Physics Amsterdam, Netherlands, K. Winter (Editor), 295, (1988),

3) C. A. Mears, S. E. Labov and A. T. Barfknecht, J. Low Temp. Phys. 93, 561, (1993)

4) W. Rothmund and A. Zehnder in Ref. 1, 196, (1991)

5) W. Rothmund and A. Zehnder, in Superconductive Particle Detectors, Torino, edited by A. Barone, (World Scientific Pub., Singapore), 52, (1987) W. Rothmund and A. Zehnder, in Low Temperature Detectors for Neutrinos and Dark Mattered., edited by L. Gonzales-Mestres and D. Perret-Gallix, (Editions Frontieres, France), 217, (1988)

6) J. J. Chang and D. J. Scalapino, Phys. Rev. B 15, 2651, (1977)

7) M. Kurakado, Nucl. Instr. and Meth. 196, 275 (1982)

8) W. Rothmund, ETH Thesis Nr. 9445, Paul Scherrer Insitut, 5232 Villigen, Switzerland, (1991)

9) A. Rothwarf and B. N. Taylor, Phys. Rev. Lett. 19, 27 (1967)

10) D. Debye, Ann. Phys. 39, 789 (1912)

11) S. B. Kaplan, C. C. Chi, D. N. Langenberg, J. J. Chang, S. Jafarey, and D. J. Scalapino, Phys. Rev. B 14, 4854 (1976)

12) H. J. Maris, Phys. Rev. B 41, 9736 (1991)

13) V. Narayanamurti, R. C. Dynes, P. Hu, H. Smith, and W. F. Brinkman, Phys. Rev. B 18, 6041 (1978)

14) Handbook of Mathematical Functions, edited by M. Abramowitz and I. A. Stegun,(Dover Pub., New York, 9th Ed. 1972)

15) Mark 14 NAG Fortran Library D02EBF 
16) A. Gabutti, K. E. Gray, G. M. Pugh, and R. Tibero, Nucl. Instr. Meth. A 306, 220 (1991)

17) M. A. Scherschel, C. W. Hagen, A. Zehnder, S. P. Zhou, and H. R. Ott, Physica C 193, 264 (1992)

18) S. Labov, E. Silver, T. Pfafman, Y. Wai, J. Beeman, F. Goulding, D. Landis, N. Madden, and E. Haller, SPIE Proc. 1344, 295, (1990)

19) C. K. Stahle, R. L. Kelley, S. H. Moseley, A. E. Szymkowiak, M. Juda, D. McCammon, J. Zhang, Physica B 194-196, 127, (1994)

20) D. Twerenbold, and A. Zehnder, J. Appl. Phys. 61, 1, (1987).

21) P. Verhoeve, N. Rando, P. Videler, A. Peacock, A. van Dordrecht, D. J. Goldie, J. M. Lumley, J. Hwelett, M. Wallis, and R. Venn, in SPIE Proc. 2283, 172, (1994)

22) C. W. C. Kaye, and T. H. Laby in "Tables of Physical and Chemical Constants", (Longman, London and New York, 14th edition, 1973)

P. H. Dederichs, H. Schober, and D. J. Sellmyer in Landolt-Börnstein, 13a,b, (Springer, Heidelberg and New York, 1981)

23) W. L. McMillan and J. M. Rowel, in Superconductivity, edited by R. D. Parks (Dekker, New York, 1969) 


\section{FIGURE CAPTIONS}

Fig. 1a-d: Distribution of quasiparticles $Q(\omega, t)$ and of phonons in the film $P_{F}(\Omega, t)$ and in the substrate $P_{S}(\Omega, t)$ as a function of their energy for different times after absorption of a $5.89 \mathrm{keV} \mathrm{Mn} \mathrm{K} \mathrm{K}_{\alpha} \mathrm{X}$-ray in Sn. The insert in Fig. 1d shows the labeling for the different distributions. The bath temperature was kept at $0.5 \mathrm{~K}$. The ordinate is enhanced from 0 to $5 \mathrm{meV}$ in Fig. $1 \mathrm{c}$ and 0 to $2 \mathrm{meV}$ in Fig. $1 \mathrm{~d}$ in order to show the distributions near the gap energy $\Delta_{b}$. The ordinate for Fig. $1 a$ and $b$ is from 0 to $E_{D}$.

Fig. 2: Time evolution of the $E_{q p>}, E_{p h}$ and $E_{p h<}$ (see Eq. (23)) in Al, Va, Ta, Nb, Sn, In, and $\mathrm{Pb}$-films and and $E_{s}$ in the substrate for $10 \mathrm{~nm}$ (left) and $200 \mathrm{~nm}$ (right) quasiparticle mean free path. For the labeling of the distribution see insert. The bath temperature was kept at $0.5 \mathrm{~K}$.

Fig. 3: Percentage of initial energy (5.89 keV) stored in $E_{>}$(see Eq. (23)) for different materials as a function of the quasiparticle mean free path for a bath temperature of $0.5 \mathrm{~K}$.

Fig. 4: Calculated $E_{>}$(see Eq. (23)) for $S n$ in function of the absorbed energy and of the mean free path of the quasiparticles in the film. The bath temperature was kept at $0.5 \mathrm{~K}$.

Fig. 5: Calculated $\mathrm{E}_{>}$(see Eq. (23)) for $\mathrm{Nb}$ in function of the absorbed energy and of the mean free path of the quasiparticles in the film. The bath temperature was kept at $0.5 \mathrm{~K}$. 


\section{TABLE CAPTIONS}

Tab. 1: Parameters used in the calculations. The Fermi energy was taken from Ref. 22. The remaining values were taken from Ref. 11, except for $V$; its values was calculated from Ref. 22 and 23.

Tab. 2: Experimental ${ }^{4), 24), 25)}$ and calculated non-linearity of $\mathrm{Sn}$ and $\mathrm{Nb}$ tunneling junctions. The non-linearity was calculated using Eq. 25. All tabulated junctions have a surface of 50 by $50 \mu \mathrm{m}^{2}$, but the length of their mean free path $\lambda$ is different. The calculations were made for 3 different $\lambda$ for the listed energies 


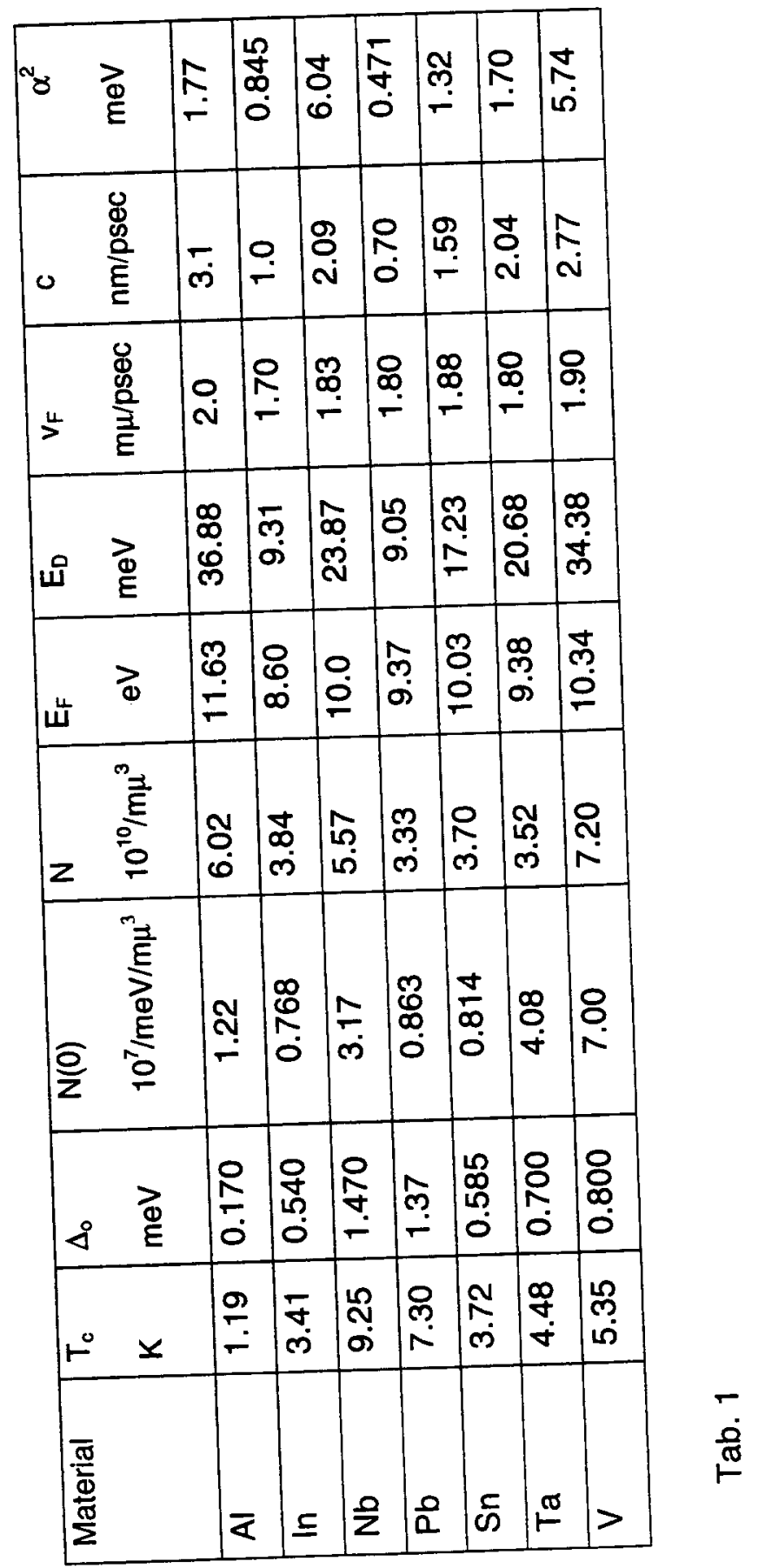




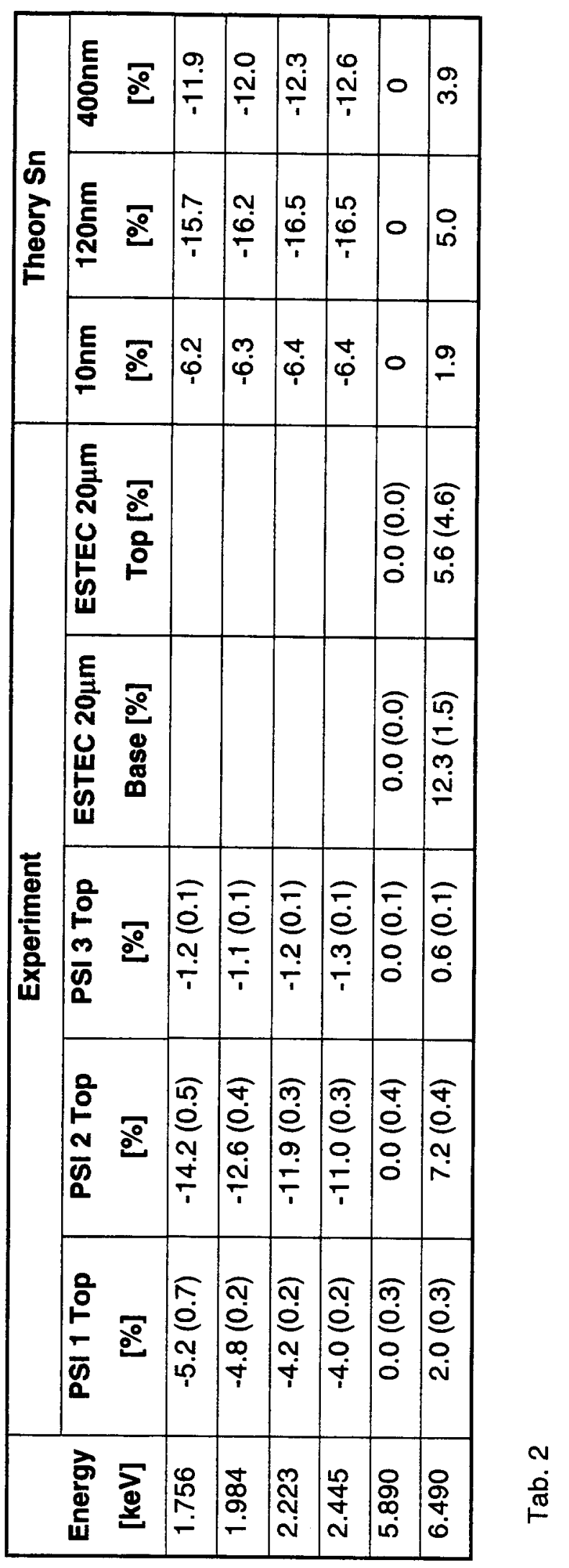



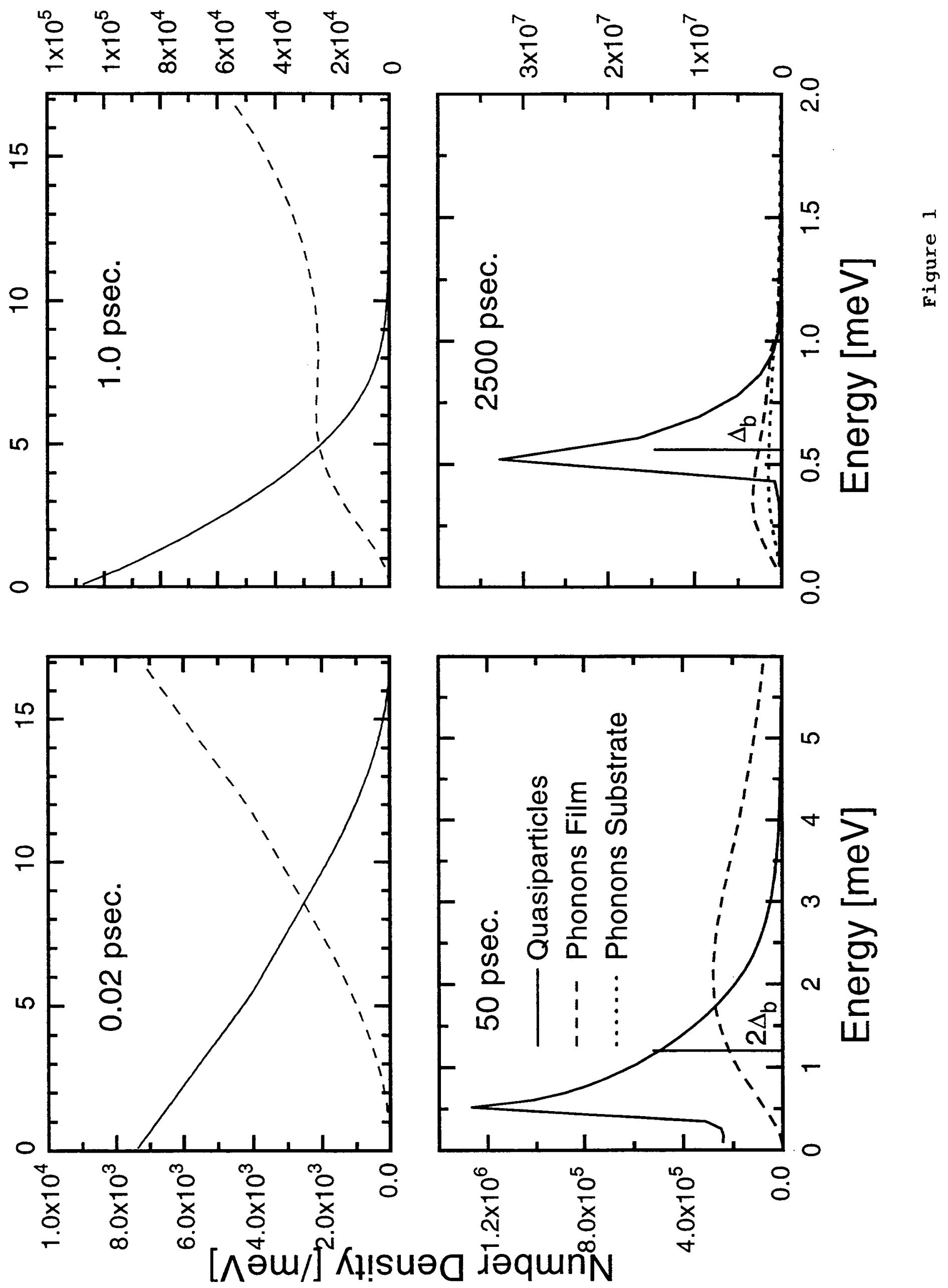


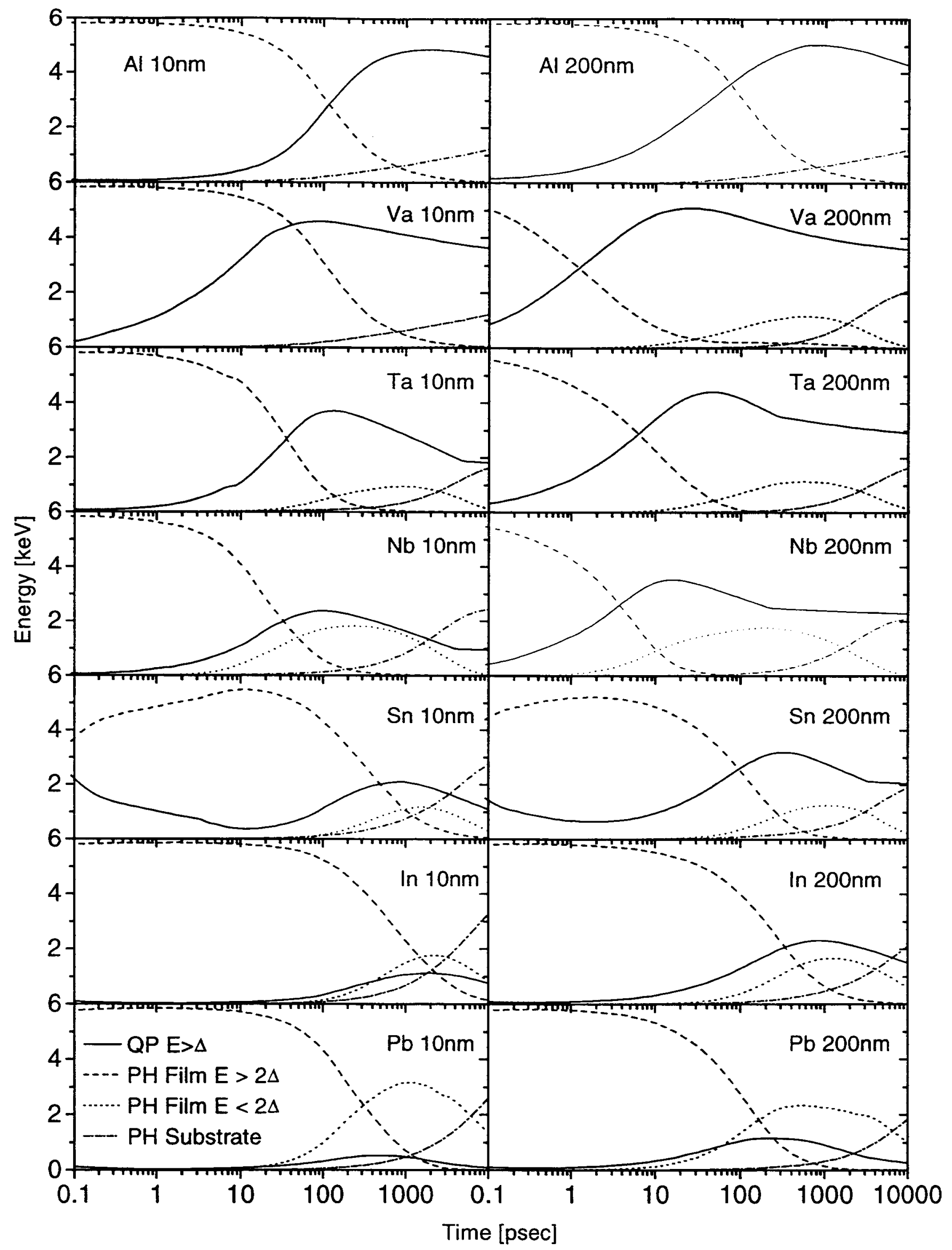

Figure 2 


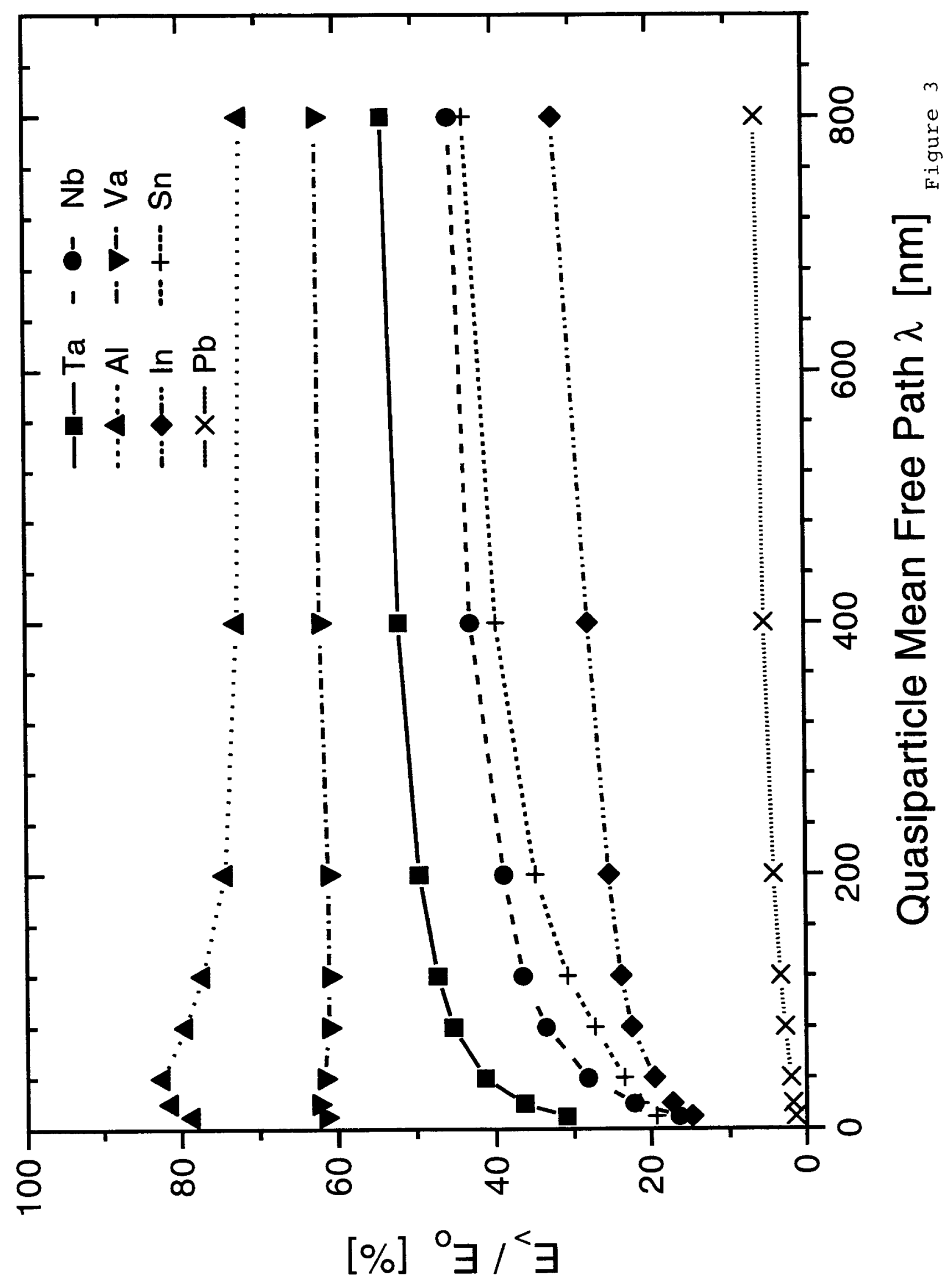




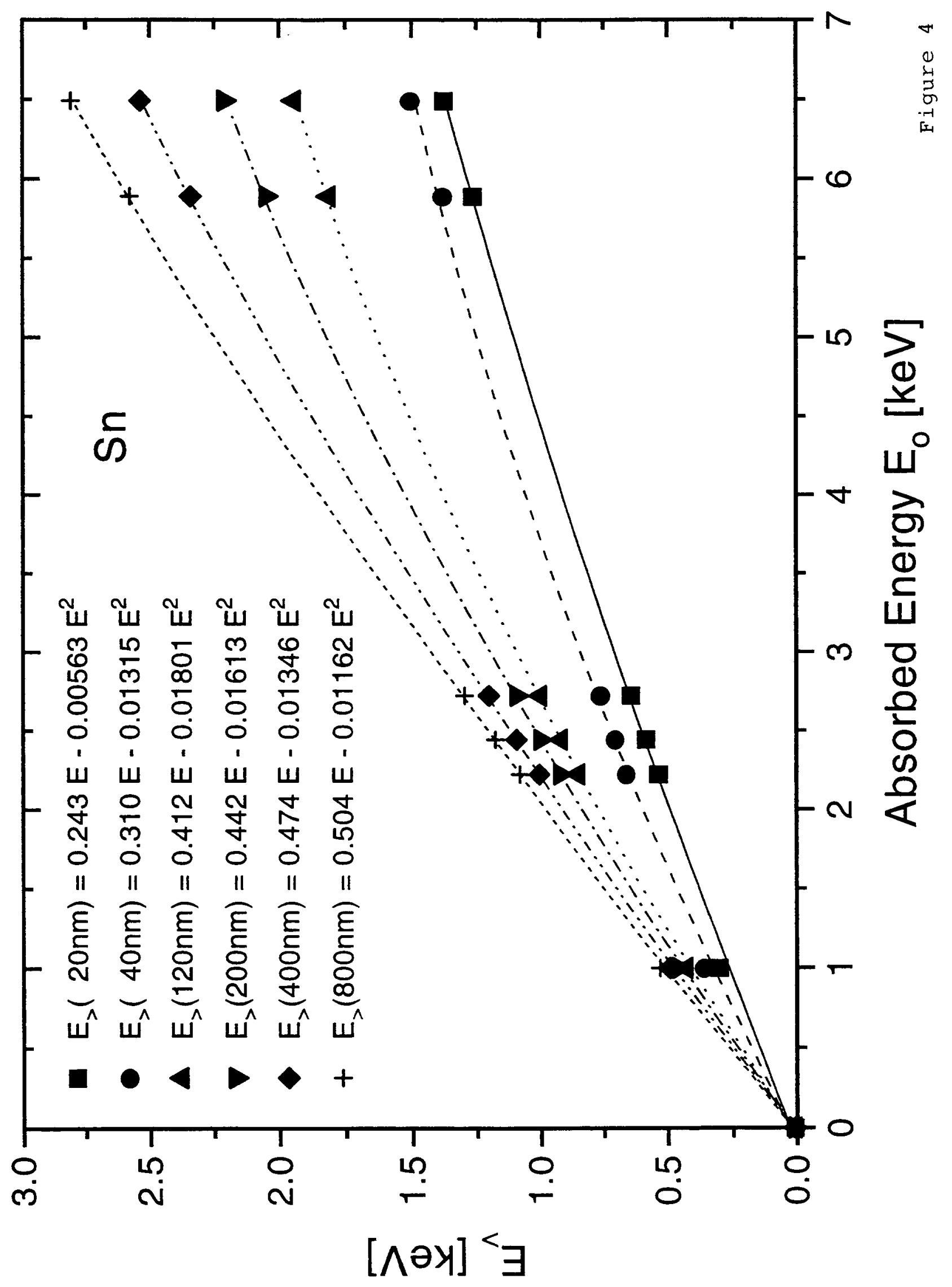




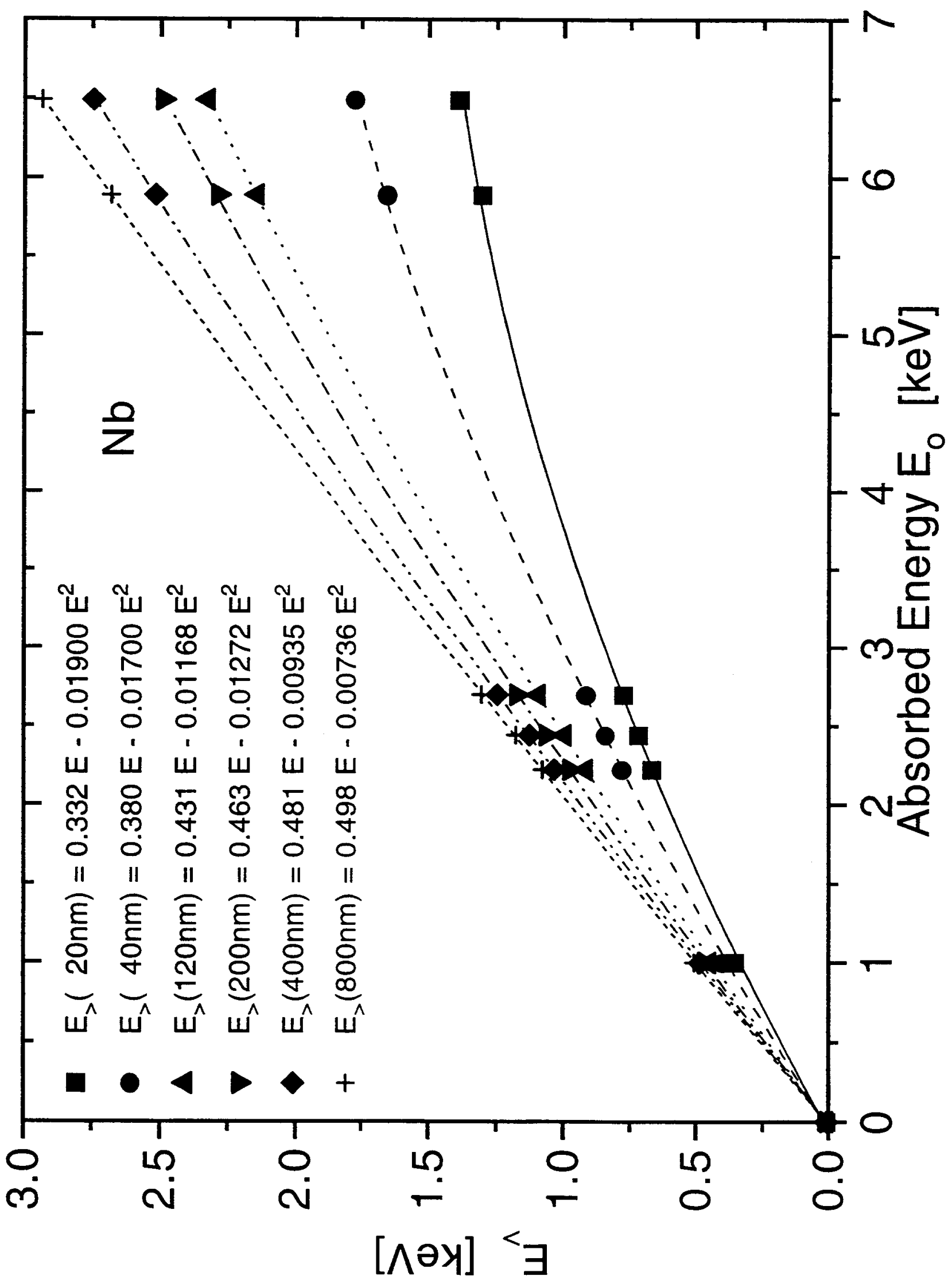

